\title{
Correspondence
}

\section{Measuring up to Pippard: ECT in practice}

DEAR SIRS

Following Pippard's recent recommendations for good ECT practice (Pippard, 1992), I would like to compare findings from our local six month audit of ECT at All Saints Hospital, a 150 bedded psychiatric hospital in inner-city Birmingham, serving a 400,000 population.

According to Pippard's scale, our premises rated five; $51 \%$ of Pippard's achieved this. The anaesthetic input includes two sessions by a regular consultant and two by a clinical assistant: this was better than $54 \%$. Medical psychiatric input included a named responsible consultant involved in training; this was better than $69 \%$. Juniors were involved in a rota system which was true in $74 \%$ of Pippard's report. It is difficult to compare his definition of quality, however. With nursing input, again comparison was as problematical, but with four trained staff it was better than most. Interestingly, it was noted that operating technicians may also have a place.

During the six months, 23 people received a course of ECT. In terms of record keeping, consent was available in 21 out of 23 patients; two forms had been lost. Timing of fits was not universally employed. Tentative figures derived from these small numbers of ECT gave a rate of 0.78 per 1000 population per annum. When tempted to judge the effectiveness of an ECT service by its relative user rate, Pippard cautions, "This is merely factual", and "should prompt enquiry as to whether ECT is being used appropriately, and the reasons for that usage". (Pippard, 1993, personal communication).

Patients' notes from 22 cases were examined for socio-demographic, diagnostic and outcome data. The mean age was 46 for males and 49 for females. Of the 22 patients, 15 were Caucasian, four AfroCaribbean and three Asian. Medication revealed some problem areas; namely, concurrent use of antiepileptics in one and benzodiazepines in four cases. In most, outcome was documented clearly. Seventeen improved, four showed no improvement, one actually deteriorated, but in two the outcome was not recorded.

In conclusion, the clinical use of ECT attained reasonable standards in most areas outlined by Pippard. However, there are areas which need improvement and further audit, including:

(a) to adopt the Royal College Guidelines recommended form (b) examine recording to include emergencies at the sister district general hospital and incorporate central recording

(c) examine the use of medications, especially benzodiazepines and anti-epileptics

(d) time fits

(e) possibly explore the use of an operating technician

(f) carry out separate review of equipment in due course

(g) study the way ECT stimulus is varied according to the patient's individual seizure threshold

(h) the adequacy of training for the doctors

(i) appropriateness of prescription and possible poor administration in treatment failures.

R. W. SOPPITT

The Archer Centre

All Saints Hospital

Birmingham B18 SSD

\section{Reference}

Pippard J. (1992) Audit of electroconvulsive treatment in two National Health Service regions. British Journal of Psychiatry, 160, 621-637.

\section{Car exhausts and suicide}

\section{Dear Sirs}

The government, in The Health of the Nation, has set the specific target of a reduction in the overall suicide rate of at least $15 \%$ by the year 2000 .

Since 1974, there has been a steady increase in the number of suicides using car exhaust fumes (from $7.7 \%$ of all suicides in England and Wales in 1974, to $28.6 \%$ in 1990). Car exhaust poisoning is now the most common method of suicide in young men aged 15-44 (Hawton, 1992), and in 1990 accounted for $12 \%$ of suicides in those aged 65 or more. This parallels the increase in the numbers of motor vehicles in the country.

There is good evidence that the availability of methods for committing suicide affects overall suicide rates (e.g. the large fall in suicides that occurred as a result of the replacement of coal gas with natural gas in the 1960s).

Although it would be impossible to restrict the access of the suicidal to motor vehicles, any reduction in the lethality of car vehicle motor exhausts would be likely to lead to a reduction in the overall suicide 